\title{
Imaging of Acute and Chronic Aortic Dissection by ${ }^{18}$ F-FDG PET/CT
}

Christian Reeps ${ }^{1}$, Jaroslav Pelisek ${ }^{1}$, Ralph A. Bundschuh ${ }^{2}$, Manuela Gurdan ${ }^{1}$, Alexander Zimmermann ${ }^{1}$, Stefan Ockert ${ }^{1}$, Martin Dobritz ${ }^{3}$, Hans-Henning Eckstein ${ }^{1}$, and Markus Essler ${ }^{2}$

${ }^{1}$ Clinic for Vascular Surgery, Klinikum-rechts-der-Isar, Technische Universität München, München, Germany; ${ }^{2}$ Clinic for Nuclear Medicine, Klinikum-rechts-der-Isar, Technische Universität München, München, Germany; and ${ }^{3}$ Institute for Radiology,

Klinikum-rechts-der-Isar, Technische Universität München, München, Germany

By conventional imaging modalities, the discrimination between acute and chronic aortic dissection (AD) for surgical risk evaluation is not possible. However, acute and chronic stable AD potentially may be distinguished by detection of reparatory hypermetabolism in the lacerated aortic wall of acute $A D$ using ${ }^{18} \mathrm{~F}-\mathrm{FDG}$ PET/CT. In this study, we analyzed the ${ }^{18} \mathrm{~F}-\mathrm{FDG}$ uptake in the aortic wall of acute and chronic stable AD. Methods: Eighteen patients with acute $(n=9)$, symptomatic progressive $(n=2)$, or known chronic stable $(n=7)$ type B AD underwent ${ }^{18} \mathrm{~F}-\mathrm{FDG}$ $\mathrm{PET} / \mathrm{CT}$. Images were analyzed qualitatively and quantitatively considering ${ }^{18} \mathrm{~F}-\mathrm{FDG}$ uptake patterns and the standardized uptake values (SUVs) of the aortic wall, dissection membrane, and luminal ${ }^{18} \mathrm{~F}-\mathrm{FDG}$ activity. The SUV ratio (maximum SUV in the aorta divided by mean SUV in the blood pool) was calculated to relativize individual luminal ${ }^{18} \mathrm{~F}-\mathrm{FDG}$ spillover effects. Results: In contrast to chronic stable $A D$, all acute or acute progressive $A D$ showed accentuated ${ }^{18} \mathrm{~F}-\mathrm{FDG}$ uptake at the injured aortic wall or dissection membrane. The maximum SUV of the dissection membrane or aortic wall was significantly higher $(P=0.02)$ in acute AD than in chronic stable AD. Thereby, SUV varied from 3.03 to 4.64 (average maximum SUV, $3.84 \pm 0.51$ ) for the dissection membrane and from 2.22 to 4.60 (average maximum SUV, $2.94 \pm 0.81$ ) for the aortic wall, with false-negative and false-positive outliers. The discrimination between acute and stable AD was improved significantly $(P<0.001)$, and false-positive or -negative outliers were eliminated, using the SUV ratio method. Conclusion: Our results indicate that ${ }^{18} \mathrm{~F}-\mathrm{FDG}$ PET/CT might be useful in differentiation of acute from chronic $A D$ in clinically unclear cases. However, larger studies are needed to confirm our preliminary results.

Key Words: PET/CT; aortic dissection; vascular imaging

J Nucl Med 2010; 51:686-691

DOI: 10.2967/jnumed.109.072298

Received Nov. 2, 2009; revision accepted Feb. 2, 2010. For correspondence or reprints contact either of the following: Hans-Henning Eckstein, Clinic for Vascular Surgery, Klinikumrechts-der-Isar, Ismaningerstrasse 22, München, Germany 81675. E-mail: gefaesschirurgie@|rz.tum.de

Markus Essler, Department of Nuclear Medicine, Klinikum-rechts-der-

Isar, Ismaningerstrasse 22, München, Germany 81675.

E-mail: markus.essler@gmx.de

COPYRIGHT @ 2010 by the Society of Nuclear Medicine, Inc.
A cute aortic dissection (AD) is a potentially lifethreatening disease and the tenth leading cause of death in Western societies. After myocardial infarction and before pulmonary embolism, AD represents the second most frequent cause of acute chest pain (1). The incidence of $\mathrm{AD}$ is about 3 in 100,000/y. Mortality rates range from 0.5 to 4 in 100,000 in national registers. However, the incidence of $\mathrm{AD}$ in necropsy studies is distinctively higher, at $1 \%-2 \%(1)$, suggesting a high number of asymptomatic, overlooked, and therefore unknown chronic ADs. Therefore, a high level of suspicion is required for the successful diagnosis because presenting symptoms of AD and chronic dissection are highly variable. In contrast to type A dissections (Stanford classification), in type B dissections, exclusively the aorta distal to the left subclavian artery is involved. In both, typically acute AD causes sudden chest pain (2), which may also be triggered by a high number of diseases. Other symptoms depend on compression and on ischemic complications. Thus, acute AD may even result in, for example, acute abdomen, paraplegia, stroke, and heart failure $(3,4)$ but may be asymptomatic in up to $5 \%$ also (1). Thus, acute AD mostly has to be diagnosed by excluding differential diagnoses. Even if $\mathrm{AD}$ is detected by conventional CT, the exact age of the lesion cannot be defined. Thereby, electrocardiogram and myocardial markers or fibrinogen and D-dimers are sometimes unhelpful for verifying or excluding acute dissection because of their low specificity (5). Consequently, in some unclear cases chronic dissection may be considered as acute and vice versa, with potentially lifethreatening therapy implications. Patients with stable dissection may be harmed, therefore, by the risk of prophylactic and potentially complicated surgery. Thus, differentiation of acute and acute secondary progressive dissections from stable chronic dissections would be of high clinical relevance. However, direct proof of acute AD would imply the detection of histopathologic changes and metabolic processes in the recently lacerated vessel wall in vivo. In analogy to findings in acute and progressive abdominal aortic aneurysm (6-12), we hypothesized 
that the detection of increased inflammatory changes by ${ }^{18}$ F-FDG PET/CT may help to discriminate acute from chronic AD.

\section{MATERIALS AND METHODS}

\section{Patients and Classification of AD}

We analyzed 18 consecutive patients with clearly acute $(n=$ 11) or chronic stable $(n=7)$ type B dissections (Stanford classification) who had agreed to participate in the study. Solely a combination of characteristic pain accompanied by hypertensive crisis, elevated serum D-dimer levels, and strictly left-sided concomitant pleural effusion on radiologic evaluation was deemed as indicative of acute AD. Nine patients presented with newly diagnosed acute symptomatic $\mathrm{AD}$, including 2 patients with acute intramural hematoma and 2 patients with acute symptomatic distal progression of known preexisting type B dissection. As a control, 7 patients (6 men and 1 woman) with asymptomatic and known stable chronic $\mathrm{AD}$ were examined. All patients with acute AD showed severe acute chest pain accompanied by hypertensive deregulation, and all had elevated D-dimer serum levels of more than $500 \mu \mathrm{g} / \mathrm{L}$. Acute pulmonary embolism and myocardial infarction had been excluded by electrocardiogram, myocardial serum markers, and pulmonary CT angiography. In addition, in acute AD CT angiography showed characteristic left-sided pleural effusion in 8 of 9 patients. The patients with acute symptomatic secondary distal progressive AD had spontaneous severe abdominal discomfort and pressure pain of the infrarenal aorta. Possible thoracic and abdominal differential diagnoses were excluded by clinical examination, laboratory analyses, ultrasound, and CT. Comparison with previous CT angiographies or medical reports substantiated suspicion of recent AD progression. All patients with acute $\mathrm{AD}$ showed no evidence of mesenteric, spinal, or limb ischemia or vasculitis.

Subsequent to emergency diagnostic evaluation, symptomatic patients were admitted to the intensive care unit for monitoring and initial medical treatment. After 3-13 d and clinical stabilization, patients with acute symptoms underwent multislice ${ }^{18} \mathrm{~F}$ FDG PET/CT including contrast-enhanced CT angiography of the aorta for dissection control.

In patients with asymptomatic chronic stable $\mathrm{AD}(n=7),{ }^{18} \mathrm{~F}-$ FDG PET/CT was on an outpatient basis. Diagnosis of chronic stable AD was confirmed by correlation of current CT angiographies with antecedent diagnostic findings not older than $1 \mathrm{y}$. Patients were excluded from the study if they had known hyperglycemia $(>150 \mathrm{mg} / \mathrm{L}$ ), renal insufficiency (serum creatinine $>1.8 \mathrm{mg} / \mathrm{dL}$ ), or an unstable cardiocirculatory condition. The study was conducted with the approval of our institutional review board, and we obtained informed consent from all the patients before their participation in the study.

\section{$\mathrm{PET} / \mathrm{CT}$}

The combined PET/CT scans were obtained 90 min after the injection of the tracer. Patients fasted at least $6 \mathrm{~h}$ before the PET examination, and the blood glucose level was less than $150 \mathrm{mg} /$ dL. Attenuation correction was based on the low-dose CT data. Patients were scanned with a Biograph 16 PET/CT (Siemens Medical Solutions) after the injection of ${ }^{18} \mathrm{~F}-\mathrm{FDG}(\sim 5 \mathrm{MBq} / \mathrm{kg}$ of body weight). The acquisition parameters for conventional CT were $120 \mathrm{kV}$ and $250 \mathrm{mAs}$, with a tube rotation speed of $0.5 \mathrm{~s}$ (table pitch, 1.2). The contrast material (iomeprol, $400 \mathrm{mg}$ of
iodine/mL; Imeron $400 \mathrm{MCT}$ [Bracco Imaging]) was injected using an automatic power injector (Stellant; MedRAD), with an injection rate of $4 \mathrm{~mL} / \mathrm{s}$. Bolus tracking was used to regulate the time at which the contrast agent arrived in the aorta. Axial slices with a 3-mm slice thickness and 3-mm reconstruction increment and a second dataset with $0.6-\mathrm{mm}$ slice thickness and $0.6-\mathrm{mm}$ reconstruction increment were reconstructed. Multiplanar reformations with a 3-mm slice thickness and maximum intensity projections with $20-\mathrm{mm}$ thickness were reconstructed in sagittal and coronal orientations. Images were analyzed using a Syngo workstation (Siemens Medical Solutions) with TrueD software (Siemens Medical Solutions); the software provides multiplanereformatted images of PET alone, CT alone, and fused PET/CT.

Imaging results were evaluated independently by a nuclear medicine specialist and a vascular surgeon and were assessed for dissection morphology and distribution of ${ }^{18} \mathrm{~F}-\mathrm{FDG}$ tracer uptake. Areas with maximum focal ${ }^{18} \mathrm{~F}-\mathrm{FDG}$ uptake were visually detected, and the maximum standardized uptake value $\left(\mathrm{SUV}_{\max }\right)$ in the dissected aortic wall or dissection membrane and in the adjacent aortic lumen was obtained by computational analyses using the TrueD software.

\section{Metabolic Definitions}

To determine the blood-pool activity, a region of interest (mean SUV in the blood pool $\left[\mathrm{SUV}_{\text {mean blood pool }}\right]$ ) was drawn over the largest luminal area possible not containing the aortic wall or dissection membrane. For the measurement of $\mathrm{SUV}_{\max }$, a region of interest corresponding to the highest ${ }^{18} \mathrm{~F}$-FDG uptake in the dissection membrane or the adjacent vessel wall was used. The SUV ratio $\left(\mathrm{SUV}_{\max }\right.$ in the aorta divided by $\left.\mathrm{SUV}_{\text {mean blood pool }}\right)$ was calculated for comparison of $\mathrm{SUV}_{\max }$ in the lacerated aortic wall or dissection membrane with the circulating luminal ${ }^{18} \mathrm{~F}-\mathrm{FDG}$ activity $\left(\mathrm{SUV}_{\text {mean blood pool }}\right)$ in the same $\mathrm{CT}$ slice.

\section{Morphologic Definitions}

Aortic pathology was assessed by analysis of typical morphologic features. The diagnosis of an AD was made in the case of an intimal dissection membrane separating the true and false aortic lumens. The diagnostic criteria of an intramural hematoma included semicircular or circular aortic wall thickening without intimal disruption exceeding $5 \mathrm{~mm}(13,14)$.

\section{Determination of D-Dimers}

Serum levels of D-dimers were measured within $24 \mathrm{~h}$ before or after the PET/CT examination. D-dimer levels were deemed to indicate acute $\mathrm{AD}$ if elevated higher than $500 \mu \mathrm{g} / \mathrm{L}$. D-dimers were determined by the BCS XP system (Siemens Medical Solutions).

\section{Statistical Analysis}

All statistical analyses were performed using SPSS for Windows (version 16.0; SPSS Inc.). Values of continuous variables were expressed as mean $\pm \mathrm{SD}$. For sample comparisons, the nonparametric Mann-Whitney $U$ test was used. Correlations between continuous variables were quantified using Spearman rank correlation coefficients.

All authors had full access to the data, take responsibility for its integrity, and have read and agreed to the article as written.

\section{RESULTS}

\section{Patients and Morphologic Imaging}

Eighteen patients with proven symptomatic acute $(n=9)$, symptomatic chronic progressive $(n=2)$, or asymptomatic 
chronic stable $\mathrm{AD}(n=7)$ underwent ${ }^{18} \mathrm{~F}-\mathrm{FDG}$ PET/CT. Sixteen of the 18 patients were men and 2 patients were women; 3 male patients had known Marfan syndrome. In acutely symptomatic patients, the diagnosis of acute $\mathrm{AD}$ was confirmed by contrast-enhanced CT angiography and synopsis of patient history, clinical course, and laboratory and imaging findings and by the exclusion of possible differential diagnoses. In patients with asymptomatic chronic stable $\mathrm{AD}$, the diagnosis was verified by comparison of recent imaging results with previous imaging results and medical reports. The age of patients at the time of clinical presentation varied from 39 to $80 \mathrm{y}$, with a mean age of $58.6 \pm 10.3 \mathrm{y}$, and was not significantly different between patients with acute symptomatic and chronic stable AD (mean, $58.2 \pm 11.7$ y vs. $58.5 \pm 7.3$ y, respectively). Maximum diameter of the aorta in patients with acute AD ranged from 32 to $67 \mathrm{~mm}$ (mean, $45.1 \mathrm{~mm}$ ) and was not significantly different from that in patients with chronic stable $\mathrm{AD}$, who had an aortic diameter ranging from 39 to $62 \mathrm{~mm}$ (mean, $49.2 \mathrm{~mm}$ ). In the 2 patients with symptomatic chronic progressive $\mathrm{AD}$, maximum aortic diameters were 40 and $77 \mathrm{~mm}$; thereby, patients showed progression of thoracic $\mathrm{AD}$ into the abdominal aorta. In symptomatic patients, ${ }^{18} \mathrm{~F}$-FDG PET/CT was performed after 2-13 d (mean, $5.7 \mathrm{~d}$ ). Further patient characteristics are shown in Table 1.

\section{Metabolic Imaging Visual Assessment}

In patients with acute $\mathrm{AD},{ }^{18} \mathrm{~F}-\mathrm{FDG} \mathrm{PET} / \mathrm{CT}$ revealed increased ${ }^{18} \mathrm{~F}$-FDG uptake in the $\mathrm{AD}$ membrane and in the

\begin{tabular}{|c|c|c|c|}
\hline Characteristic & $\begin{array}{l}\text { Acute } \\
\text { AD }\end{array}$ & $\begin{array}{c}\text { Chronic } \\
\text { progressive } \\
\text { AD }\end{array}$ & $\begin{array}{c}\text { Chronic } \\
\text { stable } \\
\text { AD }\end{array}$ \\
\hline $\mathrm{M} / \mathrm{F}(n)$ & $8 / 1$ & $2 / 0$ & $6 / 1$ \\
\hline $\begin{array}{l}\text { AD/intramural } \\
\text { hematoma }(n)\end{array}$ & $7 / 2$ & $2 /-$ & $7 /-$ \\
\hline \multicolumn{4}{|l|}{ Age (y) } \\
\hline Mean & 66.6 & 47.0 & 58.5 \\
\hline Range & $39-80$ & $41-53$ & $51-68$ \\
\hline \multicolumn{4}{|l|}{ Aortic diameter (mm) } \\
\hline Mean & 45.1 & 58.5 & 49.2 \\
\hline Range & $32-67$ & $40-77$ & $39-62$ \\
\hline $\begin{array}{l}\text { Marfan syndrome } \\
\quad(\text { yes/no) (n) }\end{array}$ & $1 / 8$ & $2 / 0$ & $0 / 6$ \\
\hline \multicolumn{4}{|l|}{ Interval to PET/CT (d) } \\
\hline Mean & 6.1 & 4 & - \\
\hline Range & $3-13$ & $2-6$ & - \\
\hline Acute pain (yes/no) (n) & $9 / 0$ & $2 / 0$ & $0 / 7$ \\
\hline $\begin{array}{l}\text { Hypertensive crisis } \\
\text { (yes/no) (n) }\end{array}$ & $9 / 0$ & $0 / 2$ & $1 / 6$ \\
\hline $\begin{array}{l}\text { Pleural effusion } \\
\text { (yes/no) }(n)\end{array}$ & $8 / 1$ & $0 / 2$ & $1 / 6$ \\
\hline $\mathrm{D}$-dimer $>500 \mu \mathrm{g} / \mathrm{L}$ & 9 & 1 & Not done \\
\hline \multicolumn{4}{|c|}{$\begin{array}{l}\text { Interval to PET/CT was defined as time from acute pain to } \\
\mathrm{PET} / \mathrm{CT} \text {. }\end{array}$} \\
\hline
\end{tabular}

adjacent aortic wall as seen by visual assessment of PET images (Fig. 1). Interestingly, acute intramural hematoma regarded as a special subgroup of type $\mathrm{B}$ dissection exhibited maximum aortic semicircular ${ }^{18} \mathrm{~F}$-FDG uptake in the lacerated aortic wall colocalized to mural hematoma (Fig. 2). In the 2 patients with symptomatic distal progressive preexisting $\mathrm{AD}$, increased ${ }^{18} \mathrm{~F}$-FDG uptake was limited to the newly injured parts of the aortic wall (data not shown). In asymptomatic patients with chronic and proven stable dissection, no noticeable ${ }^{18} \mathrm{~F}-\mathrm{FDG}$ uptake in the injured wall, compared with the surrounding tissue, was detected (Fig. 3).

\section{Quantitative Assessment of ${ }^{18}$ F-FDG Uptake}

$\mathrm{SUV}_{\max }$ was determined for each patient in the area in the dissection membrane or aortic wall with the highest visible ${ }^{18} \mathrm{~F}$-FDG uptake. Higher maximum ${ }^{18} \mathrm{~F}$-FDG uptake in the area of the dissection was found in patients with acute (average $\mathrm{SUV}_{\max }, 3.84 \pm 0.51$; range, 3.03-4.64) or symptomatic progressive chronic $\mathrm{AD}\left(n=2 ; \mathrm{SUV}_{\max }, 5.50\right.$ and 3.53 , respectively) than in patients with chronic stable $\mathrm{AD}$ (average $\mathrm{SUV}_{\max }, 2.94 \pm 0.81$; range: 2.22-4.60). As shown in Figure 4A, the difference between the $\mathrm{SUV}_{\max }$ of acute $\mathrm{AD}(n=9)$ and chronic stable $\mathrm{AD}(n=7)$ was statistically significant $(P=0.02)$. As demonstrated in Figure 4B, if solely $\mathrm{SUV}_{\max }$ was considered for the evaluation then falsenegative and false-positive outliers were found-outliers potentially caused by individual luminal spillover effects. Therefore, the ratio of $\mathrm{SUV}_{\max }$ in the $\mathrm{AD}$ wall or dissection membrane to $\mathrm{SUV}_{\text {mean blood pool }}$ was calculated to reduce these individual spillover effects. The diagnostic accuracy in the discrimination of acute (average SUV ratio, $2.20 \pm 0.48$; range, 1.62-3.09) and chronic stable $\mathrm{AD}$ (average SUV ratio, $1.24 \pm 0.28$; range, $0.82-1.50$ ) was significantly improved $(P<0.001)$ (Fig. 4C) using the SUV ratio. In addition, falsepositive or false-negative outliers considering all patients, including symptomatic progressive AD patients (SUV ratio, 2.78 and 2.21), were eliminated (Fig. 4D).

\section{DISCUSSION}

Acute AD is a life-threatening emergency, with a high number of potential differential diagnoses. So far, even advanced imaging modalities, including CT and transesophageal echocardiography, fail in the differentiation of acute and chronic dissection. Also, laboratory parameters such as fibrinogen and D-dimers are unspecific and not always effective in determining the age of the $\mathrm{AD}$. Therefore, direct proof or exclusion of acute or stable chronic $\mathrm{AD}$ by a new imaging modality would be helpful in clinically unclear cases for answering specific clinical problems and for risk stratification of AD.

In this study, the putative role of metabolic imaging by ${ }^{18} \mathrm{~F}-\mathrm{FDG}$ PET/CT for differentiation of acute, secondary progressive, and stable chronic dissection was analyzed. The combined anatomic and metabolic information of ${ }^{18} \mathrm{~F}-$ FDG PET/CT allowed an exact localization of enhanced 


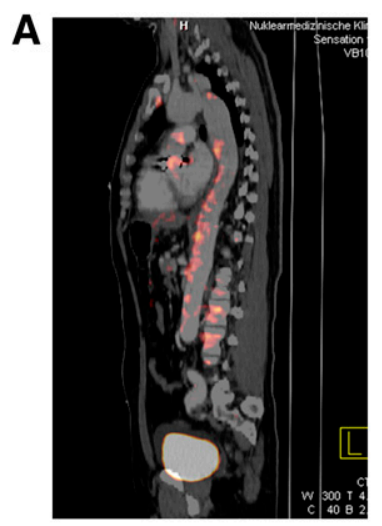

B
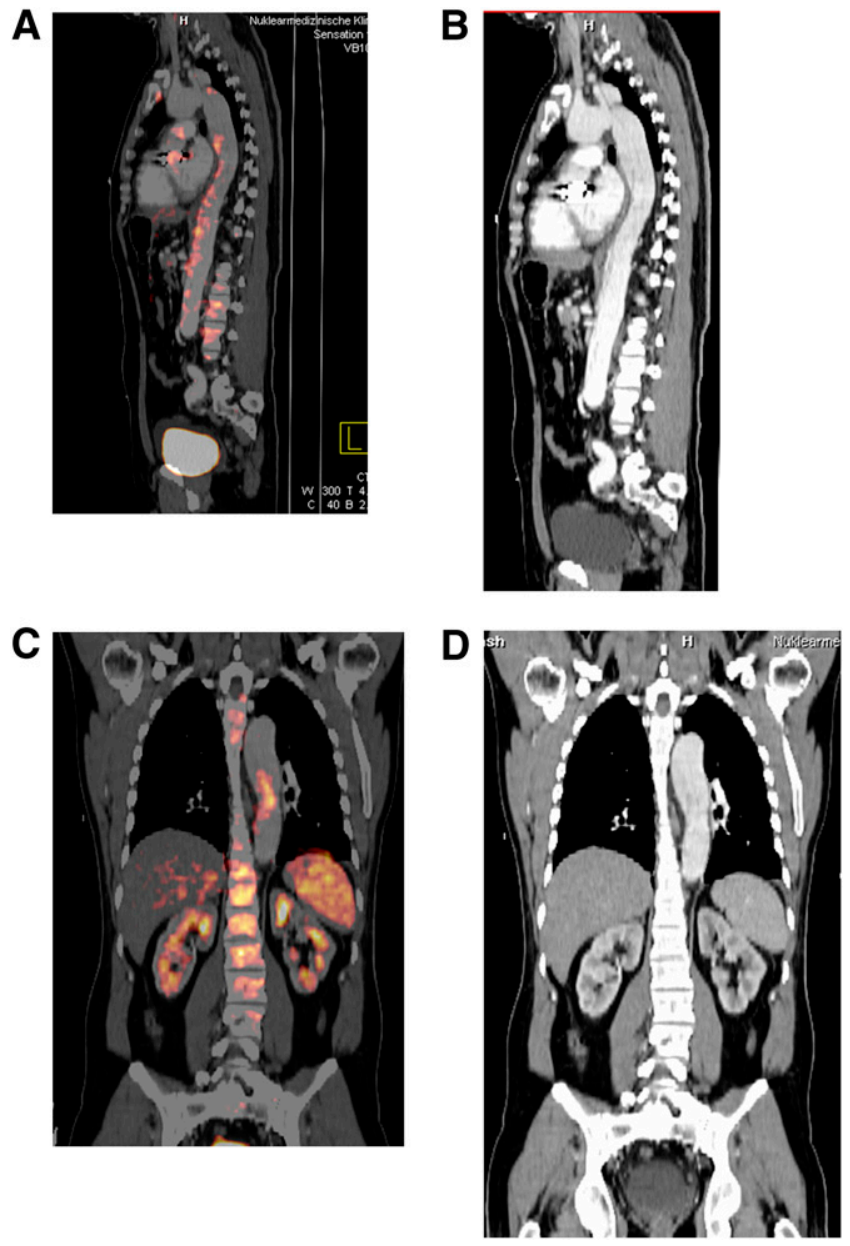

FIGURE 1. Images of 37-y-old man with acute type B dissection of aorta: sagittal (A) and coronal (C) PET/CT images and the corresponding sagittal (B) and coronal (D) nonfused CT images.
${ }^{18}$ F-FDG uptake in the aortic wall and dissection membrane. Our results suggest that acute dissection of the aortic wall leads to elevated metabolic activity in fresh lacerated segments of the aortic wall, whereas stable chronic AD did not show increased ${ }^{18} \mathrm{~F}$-FDG uptake. In contrast to chronic stable AD, patients with acute intramural hematoma and secondary progressive chronic $\mathrm{AD}$ showed also elevated glucose metabolism in the aortic vessel wall. Therefore, increased ${ }^{18} \mathrm{~F}$-FDG uptake in the aortic wall seems to correlate with acute injury and its repair, and ${ }^{18} \mathrm{~F}-\mathrm{FDG}$ PET/CT may contribute to differentiation of acute and chronic AD in clinically unclear cases.

So far, the underlying pathologic mechanisms leading to increased glucose metabolism in acute $\mathrm{AD}$ are not clear. It seems plausible, however, that the acute vascular injury may induce repair mechanisms leading to accumulation of glycolytic active cells such as macrophages and activated myofibrinocytes in the vessel wall, enhancing ${ }^{18} \mathrm{~F}-\mathrm{FDG}$ uptake. Compared with acute $\mathrm{AD}$, in chronic stable dissections there may be metabolically less active cells, such as fibroblasts, with low tissue density, forming scar tissue. This possibility agrees with recent histopathologic findings in abdominal aortic aneurysm showing a correlation between enhanced ${ }^{18} \mathrm{~F}-\mathrm{FDG}$ uptake in the aortic wall and inflammatory reactions and macrophage invasion $(7,8)$. Further studies are needed to prove these hypotheses for acute and progressive AD. Nevertheless, in acute AD it is difficult to obtain material for histologic analysis because, currently, surgical management of acute complicated $\mathrm{AD}$, type $\mathrm{B}$, is a domain of endovascular repair.

It was reported recently (12) that ${ }^{18} \mathrm{~F}-\mathrm{FDG}$ uptake in the aorta in a subgroup of patients with acute symptomatic $A D$ is correlated with a dismal prognosis. Our study corroborates the finding that ${ }^{18} \mathrm{~F}-\mathrm{FDG}$ uptake in the aorta is

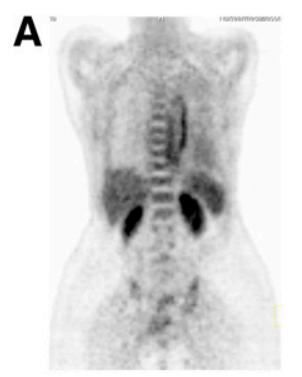

D

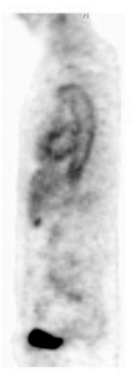

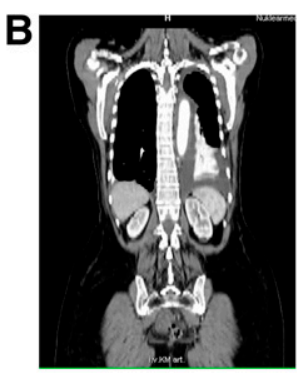

E

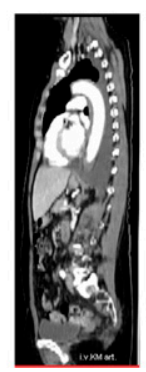

C
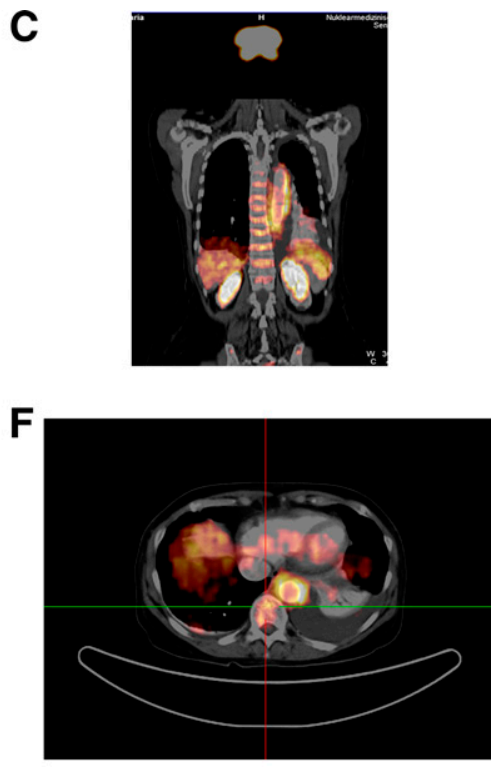

FIGURE 2. Images of 65-y-old woman with acute intramural hematoma: coronal (A) and sagittal (D) PET images, coronal (B) and sagittal (E) CT images, and coronal $(\mathrm{C})$ and axial (F) PET/CT images. 

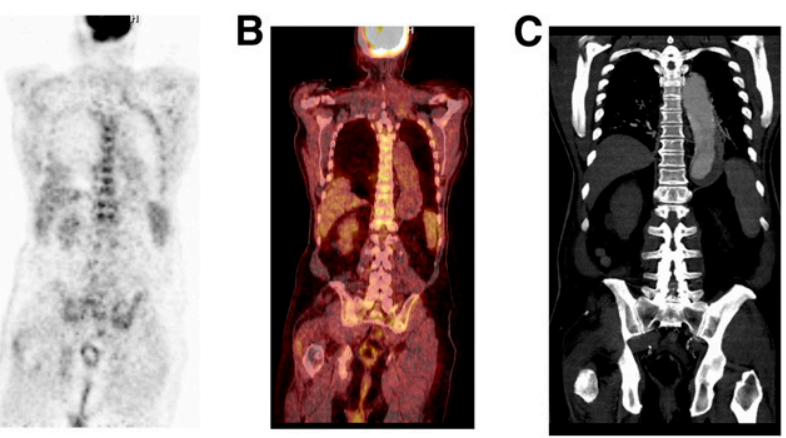

FIGURE 3. Images of 65-y-old man with chronic, stable dissection of aorta: coronal PET (A), fused PET/CT (B), and CT (C) images. No specific ${ }^{18}$ F-FDG uptake is detected.

increased in patients with acute $\mathrm{AD}$, but in our patient collective ${ }^{18} \mathrm{~F}$-FDG uptake was present in all patients with acute AD. This discrepancy may be explained by the longer interval between acute onset of $\mathrm{AD}$ and $\mathrm{PET} / \mathrm{CT}$ in the present study. Proliferative and exudative repair mechanisms in the injured aortic wall and the resulting increased glucose metabolism may be more pronounced several days after the onset of the symptoms than after $24 \mathrm{~h}$. Therefore, the time course of increased ${ }^{18} \mathrm{~F}-\mathrm{FDG}$ uptake after acute injury of the aorta must be investigated. On the other hand, inclusion criteria for the diagnosis of acute, in contrast to stable, AD were not exactly described by Kuehl et al. (12). As discussed by these authors, in this study population
D-dimer levels were frequently not indicative of acute $\mathrm{AD}$, suggesting a high proportion of preexisting chronic AD in these patients. In comparison, in our study all patients with acute $\mathrm{AD}$ showed elevated $\mathrm{D}$-dimer levels accompanied by characteristic clinical and radiologic signs.

Moreover, we analyzed the quantitative ${ }^{18} \mathrm{~F}$-FDG uptake and compared patients with acute and chronic stable $\mathrm{AD}$, whereas the study by Kuehl et al. (12) was a primarily qualitative analysis of PET images. We found that $\mathrm{SUV}_{\max }$ measured in the vessel wall is influenced by spillover effects due to activity present in the blood pool, leading to decreased diagnostic accuracy of $\mathrm{SUV}_{\max }$. As demonstrated, considering the individual circulating ${ }^{18} \mathrm{~F}-\mathrm{FDG}$ activity, these luminal spillover effects could be eliminated by the introduction of SUV ratio.

Therefore, ${ }^{18}$ F-FDG PET/CT may be helpful in the diagnosis and risk stratification of $\mathrm{AD}$ patients. However, the diagnostic value of this new imaging modality has to be corroborated by further studies with a higher number of patients. Moreover, our results may also be influenced by patient selection: in-room times of 30-45 min are required for patients undergoing PET/CT. This lengthy time leads to a preselection bias, because clinically unstable patients or patients requiring immediate therapy had to be excluded from the study. However, future development of more sensitive PET detectors will decrease the time per bed position and may result in in-room times of 15 min or less, facilitating PET examination of hemodynamically unstable patients.

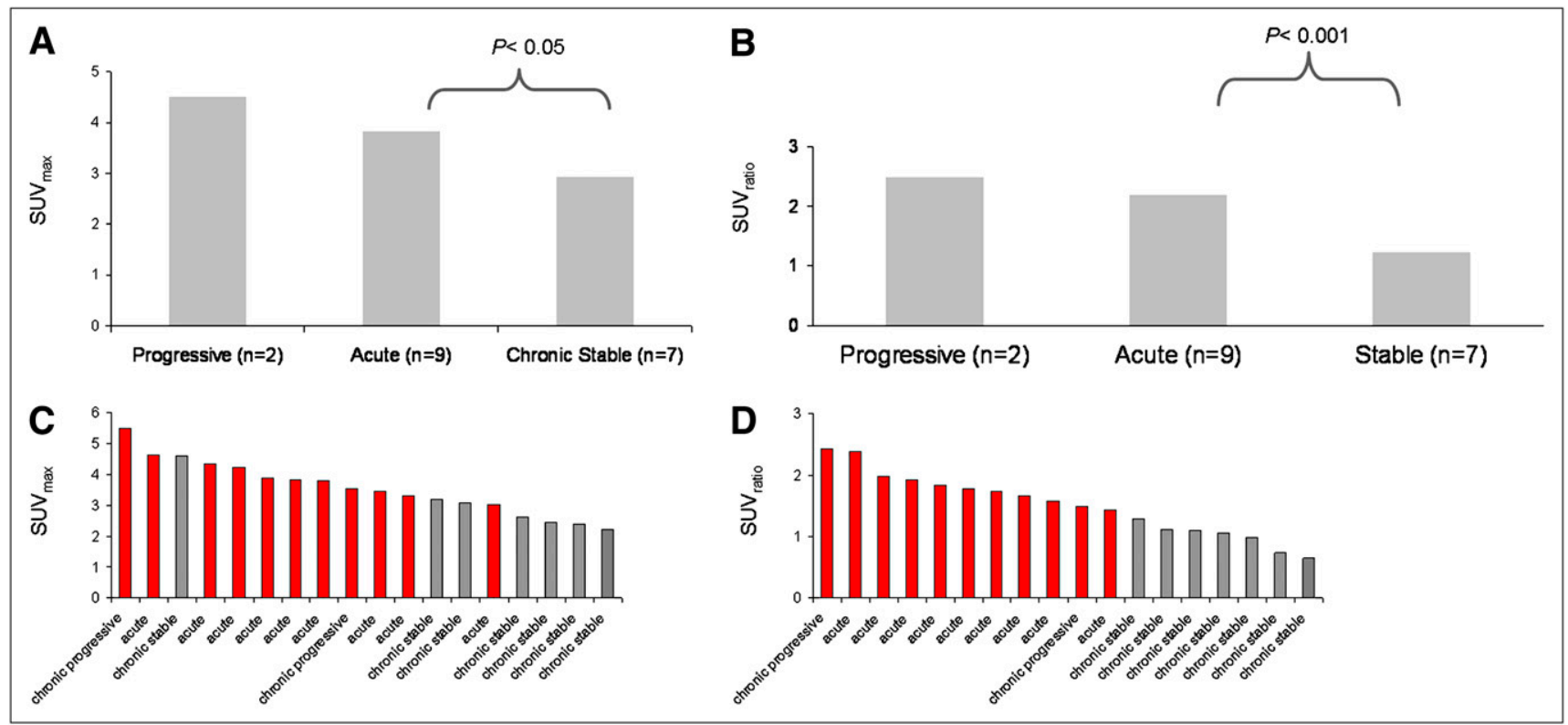

FIGURE 4. Quantitative analysis of ${ }^{18} \mathrm{~F}-\mathrm{FDG}$ uptake in vessel wall of patients with dissection of aorta. (A) $S U V_{\text {max }}$ in vessel wall adjacent to dissection. SUV $\max$ of patients with acute AD was significantly higher than that of stable patients $(P<0.05)$. (B) Ratio

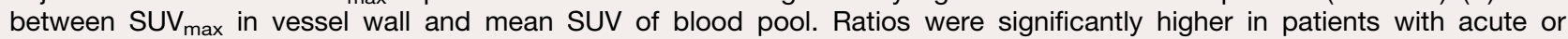
progressive $A D$ than in patients with stable $A D(P<0.001)$. (C) Individual SUV $\mathrm{V}_{\text {max }}$ of all patients. Red bars $=$ acute or chronic progressive AD; gray bars = stable AD. (D) Individual SUV ratio of all patients. Red bars = acute or chronic progressive AD; gray bars $=$ stable $A D$. 


\section{CONCLUSION}

Patients with acute AD show a higher ${ }^{18}$ F-FDG uptake at the dissection membrane than do patients with stable AD. In clinically unclear cases, PET/CT may help determine the age of an $\mathrm{AD}$, the degree of risk, and the need for surgery.

\section{REFERENCES}

1. Ince $\mathrm{H}$, Nienaber $\mathrm{CA}$. Diagnosis and management of patients with aortic dissection. Heart. 2007;93:266-270.

2. Tsai TT, Nienaber CA, Eagle KA. Acute aortic syndromes. Circulation. 2005; 112:3802-3813.

3. Urbania TH, Hope MD, Huffaker SD, et al. Role of computed tomography in the evaluation of acute chest pain. J Cardiovasc Comput Tomogr. 2009;3(1 suppl): S13-S22.

4. Lin LF, Tung JN. An uncommon cause of acute abdomen: aortic dissection complicated by superior mesenteric artery occlusion. J Dig Dis. 2009;10:74-75.

5. Eggebrecht $\mathrm{H}$, Naber CK, Bruch $\mathrm{C}$, et al. Value of plasma fibrin D-dimers for detection of acute aortic dissection. J Am Coll Cardiol. 2004;44:804-809.

6. Rudd JH, Myers KS, Bansilal S, et al. $\left[{ }^{18} \mathrm{~F}\right]$ fluorodeoxyglucose positron emission tomography imaging of atherosclerotic plaque inflammation is highly reproducible: implications for atherosclerosis therapy trials. J Am Coll Cardiol. 2007;50:892-896.
7. Reeps C, Essler M, Pelisek J, et al. Increased $\left[{ }^{18} \mathrm{~F}\right]$ fluorodeoxyglucose uptake in abdominal aortic aneurysms in positron emission/computed tomography is associated with inflammation, aortic wall instability, and acute symptoms. $J$ Vasc Surg. 2008;48:417-423.

8. Reeps C, Gee MW, Maier A, et al. Glucose metabolism in the vessel wall correlates with mechanical instability and inflammatory changes in a patient with a growing aneurysm of the abdominal aorta. Circ Cardiovasc Imaging. 2009;2:507-509.

9. Sakalihasan N, Hustinx R, Limet R. Contribution of PET scanning to the evaluation of abdominal aortic aneurysm. Semin Vasc Surg. 2004;17:144153.

10. Takahashi M, Momose T, Kameyama M, Ohtomo K. Abnormal accumulation of $\left[{ }^{18} \mathrm{~F}\right]$ fluorodeoxyglucose in the aortic wall related to inflammatory changes: three case reports. Ann Nucl Med. 2006;5:361-364.

11. Ryan A, McCook B, Sholosh B, et al. Acute intramural hematoma of the aorta as a cause of positive FDG PET/CT. Clin Nucl Med. 2007;32:729731.

12. Kuehl H, Eggebrecht H, Boes T, et al. Detection of inflammation in patients with acute aortic syndrome: comparison of FDG-PET/CT imaging and serological markers of inflammation. Heart. 2008;94:1472-1477.

13. Metser U, Miller E, Lerman H, Even-Sapir E. Benign nonphysiologic lesions with increased $\left[{ }^{18} \mathrm{~F}\right]-\mathrm{FDG}$ uptake on PET/CT: characterization and incidence. AJR. 2007;189:1203-1210.

14. Manghat NE, Morgan-Hughes GJ, Roobottom CA. Multi-detector row computed tomography: imaging in acute aortic syndrome. Clin Radiol. 2005;60:12561267. 